\title{
SIMULTANEOUS BIDIRECTIONAL PAM-6 WIRED LINK WITH ADAPTIVE PRE-EMPHASIS AND TRELLIS CODING
}

\author{
Ming-ta Hsieh and Gerald E. Sobelman \\ Department of Electrical and Computer Engineering \\ University of Minnesota \\ 200 Union Street S.E., Minneapolis, MN 55455 USA
}

\begin{abstract}
This paper presents a novel design for a simultaneous bidirectional multilevel wireline transmission system with adaptive pre-emphasis and trellis coded modulation. The incorporation of a trellis encoder and a Viterbi decoder provides coding gain to offset the loss of SNR caused by the multilevel pulse amplitude modulation. Furthermore, the feedback loop inherent in a simultaneous bidirectional link provides a natural opportunity to use adaptive pre-emphasis to compensate for channel characteristics. Our simulation results demonstrate the effectiveness of this approach.
\end{abstract}

\section{INTRODUCTION}

Modern semiconductor technology allows for the design of integrated circuits operating with on-chip clock frequencies easily beyond a gigahertz. However, the overall performance of a system depends not only on the speed of on-chip computations but also the throughput of the $\mathrm{I} / \mathrm{O}$ and bandwidth of the transmission channel. The pin-count limits and maximum achievable I/O throughput and transmission channel bandwidth have proven to be major challenges to overall system performance. The need for higher total I/O throughput and channel bandwidth have led several researchers to study simultaneous bidirectional signaling [1-4] and multilevel signaling [5-8], respectively. For a given total I/O throughput and transmission channel bandwidth, both the simultaneous bidirectional transmission and multilevel signaling techniques offer higher timing margin but lower voltage margin. [9-10] Therefore, simultaneous bidirectional multilevel transmission is most suitable for low loss or short link communications. Recently, industry has looked at the possible combination of multilevel signaling and simultaneous bidirectional transmission for extending the bandwidth of today's high-speed serial backplanes without adaptive preemphasis and coding modulation. [11]

Bidirectional transmission is attractive because of the capability of simultaneously sending and receiving data across the same transmission channel. It doubles the effective throughput for a given pin-count compared to the traditional unidirectional transmission. On the other hand, the multilevel signaling is also attractive because each data symbol carries $\log _{2}(\mathrm{~N})$ bits of information compared to 1 bit in a binary signaling, where $\mathrm{N}$ is the number of levels. The low voltage margin in multilevel signaling can be ameliorated by the coding gain from encoder and decoder. [12-15]

Figure 1 shows the basic circuit diagram for simultaneous bidirectional multilevel transmission with trellis encoding and Viterbi decoding. Replica-Diver.L and Replica-Driver.R have the same characteristics as Driver.L and Driver.R, respectively. Therefore, echo cancellation can completely remove its own side driver outgoing signal and extract the received signal, $\mathrm{V}_{\mathrm{EL}}$ or $\mathrm{V}_{\mathrm{ER}}$. In the figure, $\mathrm{T}_{\mathrm{O}}$ represents the delay time for the wave traveling through the transmission channel. For simplicity, we have chosen a trellis encoder based on a rate $2 / 3$ (8-state) code from [15] and map a 5-bit binary input into a 2-dimensional, 6level pulse amplitude modulated (2D PAM-6) signal, as shown in Figure 2. The feedback loop inherent in the bidirectional link provides a natural opportunity for incorporating adaptive preemphasis into the design. The use of an adaptive pre-emphasis feature provides transmission channel loss compensation. The advantages of using a transmitter pre-emphasis compared to receiver equalization are lower power consumption, superior performance and interoperability [16]. However, pre-emphasis is not preferred over receiver equalization if cross-talk is one of major limitations in the transmission data degradation. [17]

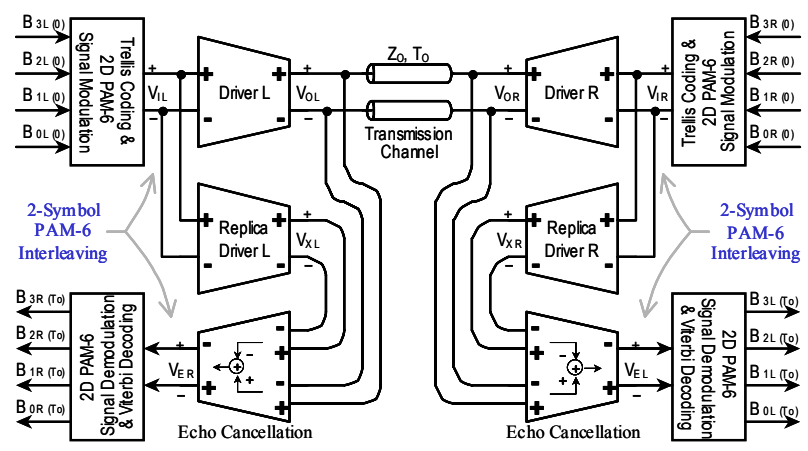

Figure 1. The basic circuit diagram of a simultaneous bidirectional multilevel transmission system.

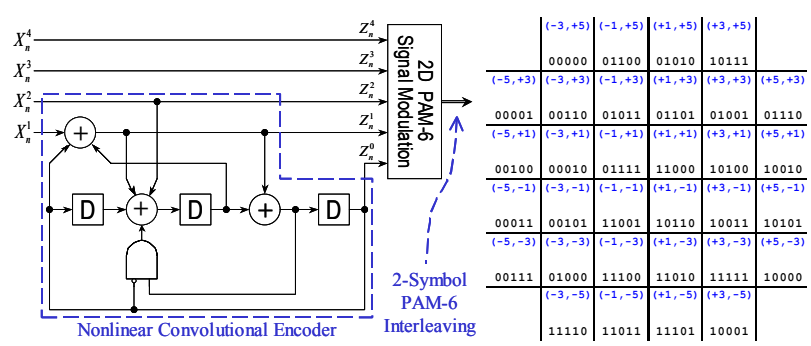

Figure 2. Encoder for a rate 2/3 (8-state) code and signal mapping of a 5-bit binary code into 2D PAM-6. 


\section{ARCHITECTURE}

Simultaneous bidirectional transmission systems can be defined as either current-mode or voltage-mode and as differential or single-ended transmission systems. A current-mode driver draws constant current though the termination load and therefore consumes more power, but it has better impedance matching and smaller reflections than a voltage-mode driver. Furthermore, a current-mode driver potentially provides higher bandwidth than a voltage-mode driver because the equivalent load on the output of the driver is $\mathrm{Zo} / 2$ and $\mathrm{Zo} \times 2$ for currentmode and voltage-mode, respectively. For the same parasitic capacitance associated with a driver output load, a current-mode driver has 4 times as much bandwidth as a voltage-mode driver. The difference between differential and single-ended signaling is that the former is immune to common-mode noise (e.g. substrate noise) but has only half of the total I/O bandwidth compared to latter. Since a high-speed simultaneous bidirectional signaling system would be sensitive to impedance mismatch and noise, we have chosen to implement a configuration based on a differential current-mode driver for our study of adaptive pre-emphasis.

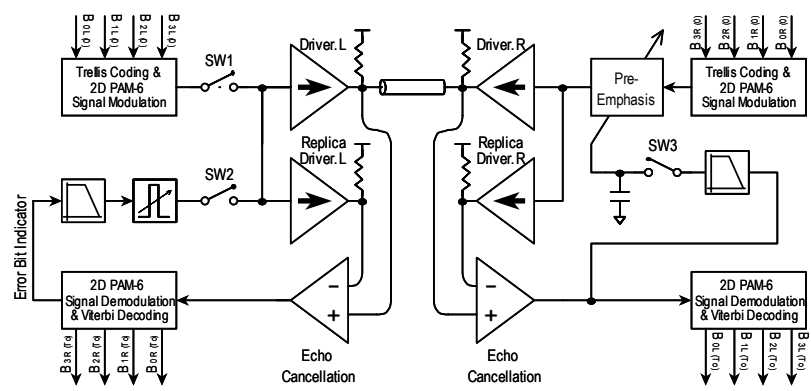

Figure 3. The proposed simultaneous bidirectional signaling system with an adaptive channel pre-emphasis (One only side of pre-emphasis is drawn).

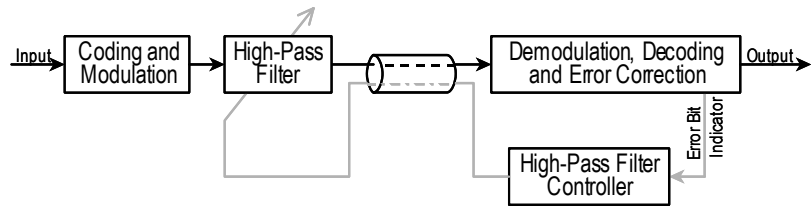

Figure 4. The conceptual circuit diagram for the adaptive channel pre-emphasis.

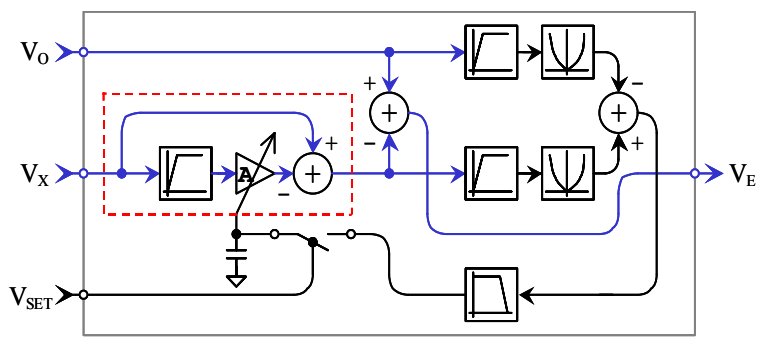

Figure 5. The basic circuit diagram of echo cancellation.

Figure 3 shows the basic circuit diagram for our proposed system. For simplicity and readability, the figure has been drawn in a single-ended mode. For comparison, Figure 4 shows the conceptual circuit diagram of an adaptive pre-emphasis scheme.
Our design approach is to first determine the degree of degradation on the received signal based on the number of error corrections during the Viterbi decoding process at a given data length Then, we generate a moderately low frequency (e.g. 1/10 the normal data rate) pulse width modulated (PWM) signal which gets fed back to the transmitting side through the existing simultaneous bidirectional link. The moderately low frequency provides minimum signal loss on the transmission channel. The PWM signal is then averaged and used to adjust the amplitude of pre-emphasis applied at the transmitter. Prior work on preemphasis to compensate for channel characteristics has been done in the context of Fast Ethernet and high-speed cable links [18-20]. However, these systems require an extra dedicated wire to carry the control signal back to the transmitter. The novelty of our approach is that we make use of the inherent bidirectional nature of the link to carry the control information without any additional wire, as well as the fact that the preemphasis is applied to signals propagating in both directions. The transmitter pre-emphasis design is based on a current-mode logic (CML) driver [21] with an adjustable 2-tap pre-emphasis. For our design, the transmitter driver pre-emphasis has been implemented with a constant current difference between driver and pre-emphasis paths so that the amplitude of the signal eye opening is kept constant.

The design of the echo cancellation circuit is shown in Figure 5 which is based on the adaptive equalizer of Ref. [19]. However, a high-pass filter is used instead of a low-pass filter before the variable gain amplifier. The justification for this is that Driver.L and Driver.R always drive I/O and the off-chip transport channel and are always more bandwidth limited than Replica-Driver.L and Replica-Driver.R of Figure 1. The circled dashed line area represents the tunable low-pass filter.

The proposed simultaneous bidirectional multilevel transmission with the adaptive pre-emphasis and coding modulation system uses the following operational sequence:

a. Run an echo cancellation initialization setup individually with the channel and all interconnects presented and the opposite side driver turned off.

b. Run an adaptive pre-emphasis initialization setup with the channel and all interconnects presented. SW1, SW2 and SW3 in Figure 3 are "OFF", "ON" and "ON" respectively.

c. Normal simultaneous bidirectional multilevel transmission can proceed after steps $a$. and $b$. If the channel or interconnects are modified, steps $a$. and $b$. have to be revisited before continuing the bidirectional transmission.

The system can also be set up so that it will automatically revisit steps a. and b. after certain time periods in order to ensure that the echo cancellation and the adaptive pre-emphasis are still operating correctly.

\section{SIMULATION RESULTS}

The proposed design has been verified with Cadence SpectreRF and Verilog-A simulators. The transmission channel loss characteristic is based on FR-4 material which is the standard glass epoxy substrate. The transmission channel trace model is extracted from Cadence Transmission Line Model Generator and the design is based on Ref. [22] with approximately $1 \mathrm{~dB}$ loss per inch at $10 \mathrm{GHz}$ [22]. Figure 6, part (1) shows simulation results for the signal loss in channel versus the signal frequency at 2", 4", 6", 8" and 10" lengths. Figure 6, part (2) shows the design information for the transmission channel trace modeling.

Figure 7, parts (1) - (2) show the uncoded 4-bit binary data with the corresponding coded and modulated PAM-6 signals. The 2- 
dimensional ( $\mathrm{X}$ and $\mathrm{Y}$ axes) PAM-6 signals shown in Figure 2 are interleaved so that each X-axis PAM-6 signal is followed by its associated Y-axis PAM-6 signal. Figure 7, parts (3) - (4) show the unidirectional PAM-6 signals at the near- and far-end, respectively. The far-end signal shows a half closed eye diagram after a 10" transmission line. Figure 7, parts (5) - (6) show the simultaneous bidirectional PAM-6 signals for 2" and 10 " of propagation, respectively. The eye-diagram shows the 11-level characteristic of the bidirectional transport channel which is due to the superposition of the 6-level signals from each side.
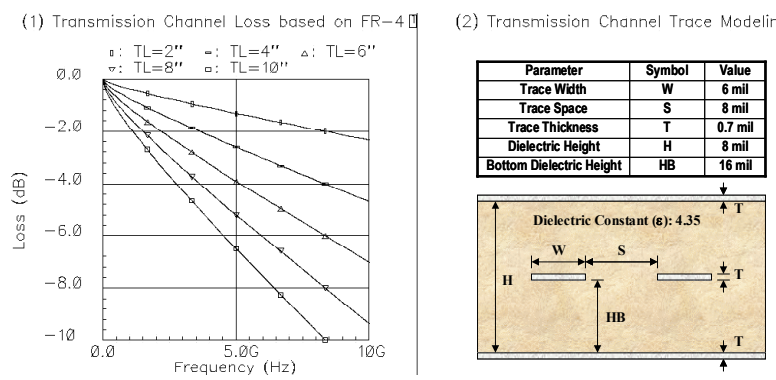

Figure 6. The transmission channel loss based on FR-4 and its trace modeling.

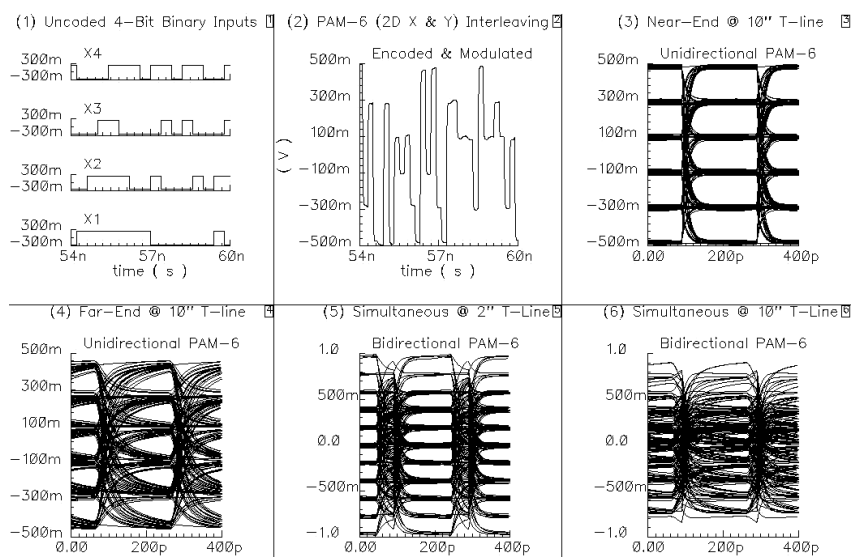

Figure 7. Simulation results for the unidirectional and simultaneous bidirectional multilevel transmission with trellis coded modulation.

Figure 8 shows the echo cancellation initialization setup simulation results for the circuits in Figure-1 and 5. The damping factor is set to be close to 0.707 for the tunable low-pass filter in echo cancellation so that the initialization time is optimized without stability concerns. The required initialization time is less than $2 \mathrm{uS}$ after reaching steady-state mode and is shown in Figure-8, part (1). The output of echo cancellation shown in Figure-8, parts (2) and (4) should be as small as possible in order to minimize the received signal degradation.

Figure 9 shows the adaptive channel pre-emphasis initialization setup simulation results for the circuit of Figure 3. The required initialization time is also less than $2 \mathrm{uS}$ after reaching steadystate mode and is shown in Figure 9, parts (1) - (2). The transient signal amplitude at the data driver output increases as the pre-emphasis voltage increases. This maintains the same difference between driver current and pre-emphasis current so that the amplitude of the maximum eye opening is kept constant. Figure 9, parts (3) - (4) show the driver input/output transient response and signal eye diagram on the transmission channel during the adaptive channel pre-emphasis initialization.

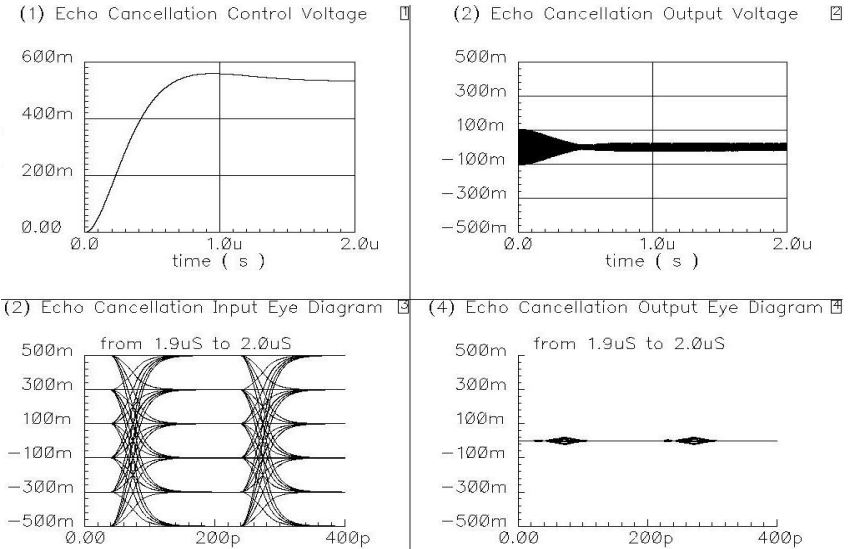

Figure 8. Simulation results for the echo cancellation during the initialization setup.
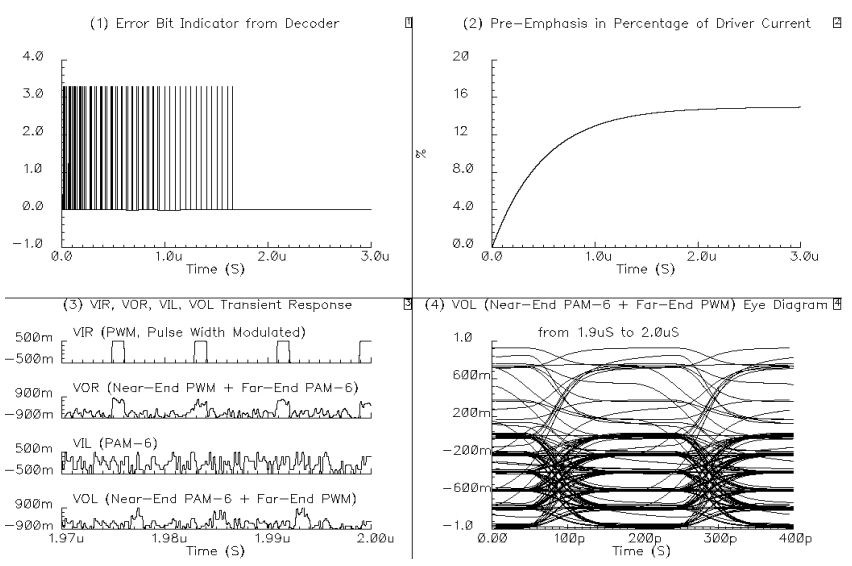

Figure 9. The adaptive channel pre-emphasis initialization setup simulation results for circuit of Figure 3.

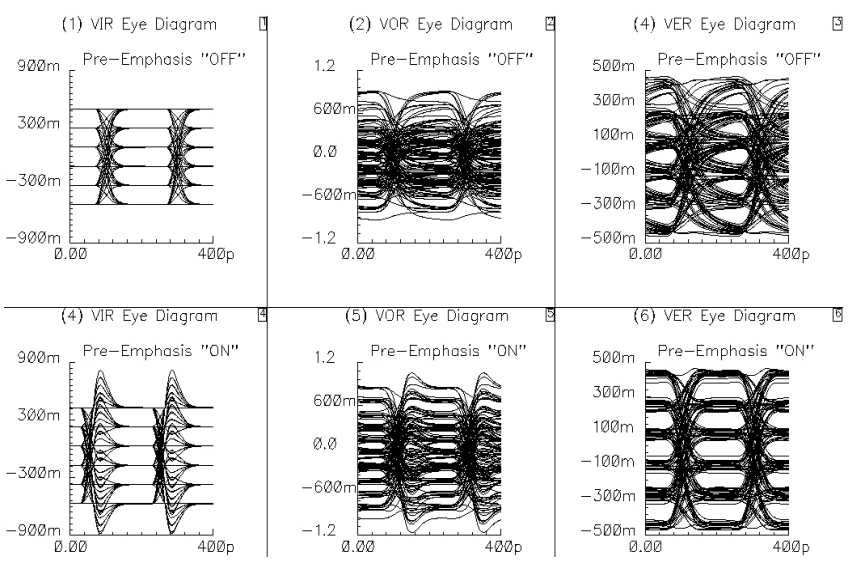

Figure 10. The simulation results for simultaneous bidirectional PAM-6 transmission with and without adaptive channel pre-emphasis and after initialization.

Figure 10 shows the simulation results for simultaneous bidirectional PAM-6 signaling with and without an adaptive channel pre-emphasis after echo cancellation and pre-emphasis are initialized. It shows that the adaptive channel pre-emphasis helps to open the eye in the VIL eye-diagram plot, which improves the rise and fall time of the received signal. 


\section{SUMMARY}

This paper presents a new design for a differential current mode simultaneous bidirectional multilevel PAM-6 transmission system for high-speed short-distance wireline communications. The differential current mode architecture provides insensitivity to common-mode noise, good impedance matching and highbandwidth performance. The simultaneous bidirectional multilevel transmission system offers higher throughput at a given pin-count and data symbol rate compared to a traditional unidirectional binary transmission system. It is most suitable for low loss or short link communications due to the low voltage margins and sensitivity to mismatched impedances. The decreased SNR inherent in multilevel modulation is offset by the coding gain of the trellis encoder and the Viterbi decoder. The adaptive channel pre-emphasis is based on a traditional receiver adaptive equalization design and utilizes the inherent feedback loop of the simultaneous bidirectional channel to automatically adjust pre-emphasis for channel loss compensation. The proposed design has been verified with Cadence SpectreRF with Verilog-A simulations. The channel loss characteristic is based on an FR-4 material model which has the standard glass epoxy substrate with approximately $1 \mathrm{~dB}$ loss per inch at $10 \mathrm{GHz}$. The simulation results show that the applied adaptive pre-emphasis for channel loss compensation can dramatically reduce the efforts needed to recover the data at each receiver side.

\section{ACKNOWLEDGMENT}

The authors would like to thank Toshiba America Electronic Components (TAEC), Inc. for the support on simulation tools and computation resources.

\section{REFERENCES}

[1] D. Cecchi, C. Hanson and C. Preuss, "A 2 GB/S High Speed Link with Differential Simultaneous Bi-Directional IO,” IEEE 2001 Custom Integrated Circuits Conference, pp. 505-508, May 2001

[2] Robert Drost, "Architecture and Design of a Simultaneously Bidirectional Single-ended High Speed Chip-to-Chip Interface," Ph.D. Thesis, Stanford University, Palo Alto, CA, Nov. 2001.

[3] D.N. de Araujo, M. Cases and N. Pham, "Design Optimization Methodology for Simultaneous Bidirectional Interface," IEEE 2001 Electrical Performance of Electronic Packaging, 2001, pp. 295-298, Oct. 2001.

[4] K.S. Canagasaby, S. Rajagopalan and S. Dabral, "Interconnect Design Challenges in Source Synchronous Simultaneous Bidirectional Links," IEEE 2002 Electrical Performance of Electronic Packaging, pp. 11-14, Oct. 2002.

[5] Ramin Farjad-Rad, Chih-Kong Ken Yang, Mark A. Horowitz and Thomas H. Lee "A 0.3- $\mu$ m CMOS 8-Gb/s 4PAM Serial Link Transceiver" IEEE Journal of Solid-State Circuits, Vol.35, No.5, pp.757-764, May 2000.

[6] Jeff Sonntag et al. "An adaptive PAM-4 $5 \mathrm{~Gb} / \mathrm{s}$ backplane transceiver in $0.25 \mu \mathrm{m}$ CMOS," IEEE Custom Integrated Circuits Conference, pp.363-366, 2002.

[7] Runsheng He, Nersi Nazari and Sehat Sutardja "A DSP based receiver for 1000BASE-T PHY," IEEE International Solid-State Circuits Conference, pp.308-309 and 458, 2001.

[8] David J. Foley and Michael P. Flynn "A low-power 8PAM serial transceiver in $0.5-\mu \mathrm{m}$ digital CMOS," IEEE Journal of Solid-State Circuits, Vol.37, No.3, pp.310-316, March 2002.

[9] D.N. de Araujo, M. Cases, N. Pham and D. Dreps, "Unidirectional vs. Simultaneous Bidirectional Source Synchro- nous Signaling," IEEE 2002 Electrical Performance of Electronic Packaging, pp. 7-10, Oct. 2002.

[10] M. Fukaishi, "GHz serial link transceiver using multiplevalued data representation," IEEE 11th International Workshop on Post Binary ULSI, May 2002

[11] L. Harrison and H. Takatori, "Extending the Life of Today's Backplanes through Simultaneous Bidirectional Transmission," DesignCon 2004

[12] A. J. Viterbi, "Error Bounds for Convolutional Codes and an Asymptotically Optimal Decoding Algorithm," IEEE Transaction on Information Theory, vol. IT-13, pp. 250269, April 1967.

[13] G. Ungerboeck, "Channel Coding with Multilevel/Phase Signals," IEEE Transactions on Information Theory, vol. 28, pp. 55-67, January 1982.

[14] G. Ungerboeck, "Trellis-coded modulation with redundant signal sets - Part I: Introduction," IEEE Communications Magazine, vol. 25, no. 2, Feb, 1987

[15] G. Ungerboeck, "Trellis-coded modulation with redundant signal sets - Part II: State of the Art," IEEE Communications Magazine, vol. 25, no. 2, Feb, 1987

[16] K. Dalmia, "High Performance Backplane Design Using the Marvell Alaska ${ }^{\mathrm{TM}}$ X Quad $3.125 \mathrm{~Gb} / \mathrm{s}$ SERDES," Marvell white paper, Nov. 2001

[17] J. Zhang and Z. Wong, "White Paper on Transmit Preemphasis and Receive Equalization," Mindspeed Technologies - A Conexant Business, Oct. 2002.

[18] X. Lin, J. Liu and J. Fonseka, "A High Speed Low-Noise Equalization Technique with Improved Bit Error Rate," IEEE 2002 International Symposium on Circuits and Systems, Volume 2, pp. 564-567, May 2002.

[19] J.N. Babanezhad, "A $3.3 \mathrm{~V}$ analog Adaptive LineEqualizer for Fast Ethernet Data Communication," IEEE 1998 Custom Integrated Circuits Conference, pp. 343-346, May 1998.

[20] K. Yoo, H. Lee and G. Han, "A Low Power CMOS Adaptive Line Equalizer for Fast Ethernet" IEEE 2002 AsiaPacific Conference on ASIC, pp. 129-132, Aug. 2002.

[21] J. Zerbe1 and etc., "Equalization and Clock Recovery for a 2.5-10Gbs 2-PAM/4-PAM Backplane Transceiver Cell", IEEE 2003 International Solid-State Circuits Conference, Feb. 2003.

[22] R. Kollipara, G Yeh, B. Chia and M. Agarwal, "Design, Modeling and Characterization of High Speed Backplane Interconnect," DesignCon 2003. 